\title{
Vers un théâtre graphique. Les jeux de l'amour et du football dans les BD de François Bégaudeau
}

\section{Camille Vorger}

\section{(2) OpenEdition \\ 1 Journals}

Édition électronique

URL : http://journals.openedition.org/edl/1247

DOI : $10.4000 /$ edl. 1247

ISSN : 2296-5084

Éditeur

Université de Lausanne

\section{Édition imprimée}

Date de publication : 15 septembre 2016

Pagination : 129-152

ISBN : 978-2-940331-49-9

ISSN : 0014-2026

\section{Référence électronique}

Camille Vorger, « Vers un théâtre graphique. Les jeux de l'amour et du football dans les BD de François Bégaudeau », Études de lettres [En ligne], 3 | 2016, mis en ligne le 15 septembre 2019, consulté le 15 décembre 2020. URL : http://journals.openedition.org/edl/1247; DOI : https://doi.org/10.4000/edl. 1247

\section{(c) Études de lettres}




\section{VERS UN THÉÂTRE GRAPHIQUE. LES JEUX DE L'AMOUR ET DU FOOTBALL DANS LES BD DE FRANÇOIS BÉGAUDEAU}

Dans cet article, notre propos est d'étudier deux BD de l'auteur contemporain François Bégaudeau en mettant en lumière la théâtralité qui leur est inhérente. Nous parlons en ce sens de "théâtre graphique» comme pendant de l'appellation "roman graphique». Notre approche se veut originale en ce qu'elle rapproche la $\mathrm{BD}$ du genre dramatique alors que la plupart des études mettent en relation le $9^{\mathrm{e}}$ art avec le $7^{\mathrm{e}}$ art. Dans cette perspective, nous analysons de nombreuses cases et planches des deux BD constituant notre corpus, en étayant notre propos d'un entretien avec leur scénariste et d'extraits du tapuscrit qu'il nous a transmis.

Viens petite fille dans mon comic strip

Viens faire des bulles, viens faire des WIP

Des CLIP! CRAP! des BANG! des VLOP! et des ZIP!

SHEBAM! POW! BLOP! WIZZ!

N'aie pas peur bébé, agrippe-toi CHRACK!

Je suis là CRASH! Ferme les yeux CRACK!

Embrasse-moi SMACK!

SHEBAM! POW! GLOP! WIZZZZZ! ${ }^{1}$

Dans la rencontre amoureuse, je rebondis sans cesse, je suis léger ${ }^{2}$.

I. Chanson créée et interprétée par Serge Gainsbourg sous le titre «Comic trip» en 1967.

2. R. Barthes, Fragments d'un discours amoureux, p. 235. 


\section{Tous les jeux sont permis}

Le jeu, dans tous les sens de ce terme, apparaît comme un fil rouge dans l'œuvre de François Bégaudeau où tous les jeux sont permis, semble$\mathrm{t}_{\mathrm{il}}{ }^{3}$, ce qui sied particulièrement à la $\mathrm{BD}$, espace de jeu pour l'auteur comme pour le lecteur: "La page est un échiquier, un jeu de go [...]. Moi la BD, j'élabore les mouvements des corps " ${ }^{4}$. Notons d'emblée que l'écriture théâtrale occupe une place nodale chez cet auteur, déjà contenue dans son roman Entre les murs (2006) qui a fait l'objet d'une adaptation cinématographique ${ }^{5}$. De fait, sa façon de traiter les dialogues, ainsi que les descriptions très précises qui les accompagnent - et qui s'apparentent parfois à une voix didascalique, nous reviendrons sur cette notion -, évoquent une esthétique théâtrale. Ainsi, d'emblée et de bout en bout, cette œuvre nous apparaît traversée par la théâtralité. Même s'il est surtout connu pour ses romans, François Bégaudeau est dramaturge, journaliste, essayiste et scénariste de BD. Nous pouvons alors émettre l'hypothèse que ces différentes voix/voies s'entremêlent et interagissent au sein d'une œuvre plurimédiale.

Comme en témoignent certaines convergences lexicales, le lien est patent entre théâtre et BD et l'œuvre de Bégaudeau - dont l'homophonie patronymique avec Godot ne nous a pas échappé et dont la parenté avec le théâtre de l'absurde reste à approfondir - semble représentative à cet égard, tout en étant emblématique du concept de «médiaculture ${ }^{6}$, non en tant qu'auteur médiatisé mais parce qu'il passe d'un medium à un autre avec dextérité et créativité. Son œuvre se caractérise en outre par une esthétique du fragment dont les vignettes d'une BD sont éminemment révélatrices. Ainsi l'écriture (au sens Barthien) est ici fortement marquée par l'oralité et le dialogisme, ancrée dans la voix et le corps qui se traduisent par des jeux de rythme - empreintes de musicalité

3. Le jeu dramatique, mais aussi sportif: le football était au cœur de son premier roman Jouer juste (2003) et a fait l'objet d'un récit de littérature jeunesse récemment réédité, intitulé L'invention du jeu (2014).

4. Article de G. Lascaut in B. Eizykman (éd.), Plates bandes à part, p. 24.

5. Le film, réalisé par Laurent Cantet, a obtenu la palme d'or au festival de Cannes en 2008.

6. Nous employons ce terme au sens de E. Maigret et E. Macé dans Penser les médiacultures avec l'idée de "décloisonner études des médias, de la culture et des représentations». 
et de corporéité dans le langage - empruntant aussi à une esthétique cinématographique et musicale. Le $9^{\mathrm{e}}$ art, auquel l'auteur s'est déjà adonné par deux fois ${ }^{7}$, apparait donc comme l'autre face, le pendant graphique de son théâtre qu'elle rejoint par une mise en scène de la parole, une projection du vocal et du corporel dans l'espace de la page, là où le théâtre projette ces voix/corps dans le double espace, sonore et visuel, de la scène.

De fait, dans la BD Mâle Occidental Contemporain (MOC), Bégaudeau semble s'inspirer de la comédie de mœurs, voire de la comédie romantique hollywoodienne: c'est une vision du jeu et du dialogue amoureux qui se dessine et s'incarne au fil des planches, celles de la BD devenant précisément le lieu où la métaphore théâtrale est filée. C’est le devenir d'une rencontre, d'un couple, qui est ici au cœur du Problème ${ }^{8}$. $M O C$, dont le titre suggère, là encore, un jeu d'homophonie ${ }^{9}$, met en abyme le jeu de la séduction, avec tout ce qu'il comporte de théâtralité et de potentialités comiques, de rebondissements. Il semble d'ailleurs que la $\mathrm{BD}$ ait été conçue d'emblée comme une forme de comédie, certaines planches s'apparentant clairement à une scène, au sens théâtral de ce terme. Il s'agit d'une première BD pour l'auteur qui semble avoir à cœur de déployer les possibles d'un tel dispositif:

Un dispositif n'est pas une forme qui sait, mais une forme qui essaie. A défaut de maîtriser un matériau, je le dispose. Je le pose devant moi, dans une configuration dont je gage qu'elle lancera des lignes de sens. J'entrelace deux fils comme Godard colle deux images: pour voir ce que ça fait ${ }^{10}$.

Dans cette communication, notre propos est de développer la théâtralité inhérente à cette $\mathrm{BD}$. En quoi ces deux media peuvent-ils entrer en dialogue et en résonance? Comment un troisième medium, voire un quatrième, à travers les expériences cinématographiques et musicales de l'auteur, ont-ils pu influer sur l'écriture de la $\mathrm{BD}$, sa poétique? Nous prolongerons cette étude en abordant la deuxième BD qu'il a

7. Une nouvelle BD est parue sous le titre Petite frappe (2014).

8. Titre d'une autre pièce de l'auteur (2008).

9. Le verbe homonyme "moque" nous rappelle, par là-même, la vocation originellement comique voire moqueuse/parodique de la BD.

Io. F. Bégaudeau, «Mort et vif», p. 8. 
scénarisée. Ainsi, après avoir exploré les relations entre théâtre et $\mathrm{BD}$, nous envisagerons $M O C$ comme une mise en scène de la rencontre amoureuse, puis Petite frappe qui traite du football et met en jeu une forme de théâtralité propre à cet univers. Pour ce faire, nous nous appuierons sur un entretien avec l'auteur et sur l'étude du scénario tapuscrit de la première $\mathrm{BD}^{11}$.

\section{La BD, théâtre graphique?}

Les BD scénarisées par Bégaudeau s'apparentent à une forme de "romans graphiques» - tout en étant nettement distinctes de l'œuvre romanesque de cet auteur - en tant que «BD d'auteur» qui déroge aux règles de la série et se distingue par un format intermédiaire entre roman et album $\mathrm{BD}^{12}$. Au-delà du rapprochement avec le roman graphique et avec le cinéma (cadrages, effets travelling, etc.), le lien avec le théâtre s'avère porteur de pistes d'analyse intéressantes et potentiellement innovantes. Aussi reprendrons-nous volontiers à notre compte, appliquée à cette œuvre, la remarque suivante de Blutch: «Contrairement à ce qu'on entend souvent, j’ai toujours trouvé la bande-dessinée plus proche du théâtre - ou du ballet - que du cinéma (...). Car la bande-dessinée, c'est l'art du dialogue - encore un point commun avec le théâtre ». ${ }^{13}$ Partant, nous proposons l'appellation de "théâtre graphique» afin d'appréhender la théâtralité inhérente à ces scénarios de $\mathrm{BD}$ qui offrent le cadre d'une véritable dramaturgie. Nous préférerons cette formule à celle d'«albumthéâtre" qu'a proposée Marie Bernanoce, essentiellement appliquée au champ du théâtre jeunesse, tout en empruntant un concept développé par cette dernière, spécialiste d'études théâtrales, à savoir celui de «voix didascalique».

II. Gracieusement transmis par l'auteur - qu'il en soit ici remercié - afin d'étayer notre propos de quelques réflexions d'ordre génétique.

I2. Sur la notion de «roman graphique», voir Baetens dans E. Maigret, M. Stefanelli (éds), La bande dessinée.

13. Cité par J. Dürrenmat, Bande dessinée et littérature, p. 100. 


\section{1. Liens a priori et rapprochements sémantiques}

Si la BD a pu être mise en relation avec l'esthétique cinématographique, son rapprochement avec le théâtre a été moins étudié, à l'exception de quelques études portant par exemple sur la transposition (ou transmédiation) du théâtre de Marivaux par Yvan Pommaux ${ }^{14}$. Or de nombreux points communs nous apparaissent, à commencer par les termes «transfuges» ${ }^{15}$ qui s'appliquent à l'un et à l'autre de ces deux champs: ainsi avons-nous pu lire dans un article de presse consacré à $M O C$ qu' «au fil des planches, la femme moderne prend corps» ${ }^{16}$. Or il va de soi que l'on retrouve ledit corps incarné "sur les planches", c'est-à-dire sur une scène théâtrale! Voilà qui ne manque pas d'attirer notre attention sur la fausse linéarité de la BD («au fil de») qui confine, dans la première BD étudiée comme dans certaines pièces de théâtre de Bégaudeau (voir infra), à la circularité: «Dans la vie, ça tourne en rond. Faudrait toujours que ça tourne en rond, une ouvre, pour être tout à fait juste" (entretien du 18/04/14) De la case à la scène, au sein de la structure même, le parallèle se confirme, le terme de "scène» étant d'ailleurs utilisé pour regrouper les cases selon l'unité de lieu.

\section{2. Observation du tapuscrit}

La structure narrative de la BD pourrait en un sens s'apparenter à celle d'une pièce de théâtre: en effet, le scénario a été composé de façon linéaire, et non avec des cases prédessinées. Notons que, dans le tapuscrit, les pages sont numérotées et les planches réparties en "mouvements", séparées par des planches-cases que l'on serait tenté de nommer «tableaux»- pour reprendre cet autre mot transfuge qui s'applique à la peinture autant qu'au théâtre - offrant une sorte de respiration visuelle à travers un espace ouvert, non compartimenté. Certains passages (les scènes de manifestations) sont d'ailleurs appelés «intermèdes" dans le tapuscrit, terme qui s'applique essentiellement au domaine des arts

I4. Collection «Rue Marivaux», voir notamment les travaux de Marie Bernanoce à ce sujet.

I5. Nous faisons ici référence au magazine culturel au sein duquel François Bégaudeau écrit des critiques cinéma et littéraires.

I6. next.liberation.fr/sexe/2013/08/27/deboires-du-male-contemporain-ou-les-fantasmes-de-begaudeau-et-oubrerie_ 927480 
du spectacle. L'ensemble est structuré par des séries de huit répliques destinées à être associées dans une même planche en une rythmique régulière: "J'étais très soucieux de m'adapter, de penser dans le support. C'est la forme qui pense» (entretien cité) ${ }^{17}$. Sur un plan génétique et épitextuel, il semble évident que la rédaction d'un scénario de BD étant peu codifiée, Bégaudeau - plus familier d'autres genres tels que le théâtre et reconnu en tant quauteur, ce qui lui confère sans doute une certaine liberté dans ses choix - a utilisé les outils méthodologiques et terminologiques à sa disposition ( $\mathrm{y}$ compris des termes relevant du champ cinématographique dans lequel il exerce comme critique), mais l'analogie nous semble aller bien au-delà de cette seule correspondance terminologique.

Il va de soi que, sur le plan du mode de réception, le public diffère assez fondamentalement de l'un à l'autre de ces deux univers: la $\mathrm{BD}$ relève de la "bibliosphère" (Bobillot ${ }^{18}$ ) et s'adresse a priori à un lectorat individuel, là où le théâtre s'adresse par essence à un collectif. Cependant, l'adresse fondamentale et les multiples points de rencontre que nous développerons ci-après nous ont confortée dans l'idée de ce rapprochement:

Dans un espace abstrait, Thomas porte loveless comme dans un rock acrobatique

Elle porte Thomas comme dans un rock acrobatique

Lui à quatre pattes et elle debout sur son dos, salue un public imaginaire

Elle à quatre pattes et lui debout sur son dos, salue un public imaginaire ${ }^{19}$.

Il y a là une forme de mise en scène avec une double adresse: le public imaginaire coïncide, en l'occurrence, avec le lectorat de cette BD qui revêt ici une dimension proprement spectaculaire. Or c'est bien une voix didascalique qui se développe dans les passages descriptifs, au sens où l'entend Marie Bernanoce dans ses recherches visant à montrer que le texte didascalique relève d'une forme discursive. De même que cette

I7. On aura reconnu ici l'allusion à la formule emblématique de Marshall Mac Luhan: "The medium is the message» (1964).

I8. Terme qui prend sens dans la réflexion de ce poète et poéticien sur poésie et medium. Voir ici www.akenaton-docks.fr/DOCKS-datas_f/collect_f/auteurs_f/B_f/ BOBILLOT_F/TXT_F/Doc(k)s-Bob.htm

I9. Extrait du tapuscrit transmis par l'auteur, p. 78 (nous soulignons). 
voix didascalique "oriente complètement la lecture et l'imaginaire de la scène ${ }^{20}$, la voix du scénariste informe la mise en images du scénario. D'une certaine façon, l'image apparaît comme une seconde voix résultant de l'appropriation et de la traduction graphique de la première ${ }^{21}$, d'où une polyphonie immanente à ce "théâtre graphique".

\section{3. Scénario et mise en scène: une polyphonie en acte}

Cette mise en scène finale (avec salutations du "public imaginaire») n'a cependant pas été mise en image telle quelle. En effet, les images théâtrales que l'auteur avait en tête ne se sont pas toujours concrétisées sous cette forme au sein des planches de la BD. La parole - en l'occurrence celle du scénariste - fait voir selon un régime de sous-détermination, là où le dessin (sur)détermine et délimite dans l'espace de la case. Le dessinateur est alors l'interprète d'un scénario dont il fait jouer (au double sens de ce terme ${ }^{22}$ ) les acteurs :

Un dessinateur de bandes dessinées fait jouer ses acteurs de papier à sa manière, il a sa propre rhétorique du cadre et ses propres conceptions en matière de mise en page et de couleur; il cumule les rôles d'éclairagiste, de costumier, de décorateur et de metteur en scène; bref, il est véritablement l'interprète, au sens le plus plein de ce terme, du scénario, il l'infléchit dans une direction où il est le seul à pouvoir aller $^{23}$.

De fait, la $\mathrm{BD}$ offre un spectacle autant qu'un récit, une représentation plus qu'une narration, et permet paradoxalement de "sortir du cadre", du despotisme d'un narrateur en donnant lieu à plusieurs voix/voies, ce qui va dans le sens de la vision démocratique de Bégaudeau, manifeste dans son œuvre à travers un souci constant de polyphonie exprimée sous diverses formes:

20. M. Bernanoce, "La didactique du texte de théâtre», p. 231.

2I. Philippe Marion parle de "graphiation» pour désigner cette forme d'énonciation graphique: http://sites.uclouvain.be/rec/index.php/rec/article/viewFile/1251/1101

22. Nous pensons ici au sens d'" avoir du jeu» (i. e. de la marge, de la liberté).

23. T. Groensteen, La bande dessinée mode d'emploi, p. 87. 
Il y a un truc qui me déplait dans le roman et Dieu sait si c'est ma forme de base où j'essaie de créer des contre-pouvoirs, c'est que le narrateur est omnipotent: c'est un monarque (entretien cité).

Ainsi la BD, à l'instar du théâtre, représente-t-elle le lieu idéal pour une mise en œuvre - en discours et en images, "en scène» donc, d'une certaine façon - de cette polyphonie.

\section{MOC ou la mise en scène de la rencontre}

2. 1. La métaphore théâtrale

Comme évoqué précédemment, la $\mathrm{BD}$ se répartit en séquences, délimitées par des tableaux (vignettes en pleine page). Notons que l'unité de lieu est prégnante sur un certain nombre de planches. La métaphore théâtrale chère à Shakespeare est présente dès l'incipit:

A un homme qui vivait là, il ne restait qu'à se tenir en bord de scène... simple spectateur aux abois. Et s'il venait à l'homme l'idée saugrenue d'entrer en scène... d'entrer dans le jeu et dans la case... il semblait qu'il en chassait immédiatement l'actrice principale (p. 3-4) ${ }^{24}$.

\section{2. La mise en espace}

On observe d'emblée une opposition entre activité (acteur ou comédien) et passivité (spectateur) : qui mène le jeu/la danse? Le narrateur se pose en spectateur, semble hors-jeu, ce qui favorise l'identification du lecteur/regardeur avec cet anti-héros au prénom neutre, "passepartout", de Thomas, sorte de Tom Pouce tel qu'il se présente sur la première de couverture (fig. 1).

24. Notons que ce texte qui correspond en fait au "récitatif» des six premières cases - réparties sur deux planches - n'est nullement délimité par un encadré mais intégré à l'image, ce qui en fait une sorte de "récitatif» au sens où l'on emploie ce terme dans l'opéra, impression qu'accentue l'usage des points de suspension soulignant la continuité. 
Fig. $1-M O C$, première de couverture. 
Cette opposition se double d'une tension entre horizontalité et verticalité, très nette à la page 3 (fig. 2) ou encore à la page 26 où Thomas est allongé sur le sol, le regard tourné vers le lecteur ("regard caméra" accentuant l'effet d'adresse) au sein d'un plan horizontal:

C'était à se demander si on était à la hauteur... ou si on était en dessous de tout?

Au-delà du jeu sur les phrasèmes (être aux pieds de, à la hauteur... ${ }^{25}$ ), il semble que l'horizontalité et le cadrage adopté tendent à renforcer la connivence avec le lecteur, son identification avec cet antihéros. Cyril Lepot a pu souligner la primeur de l'horizontalité dans la BD, du fait de

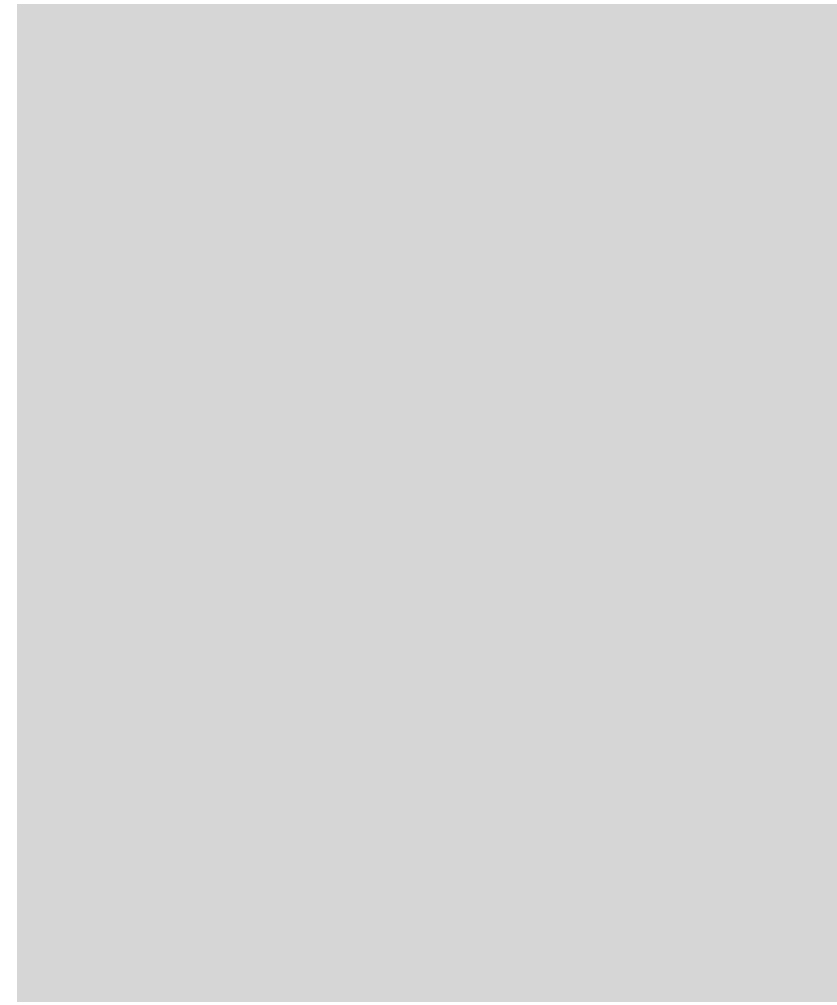

Fig. $2-M O C$, p. 3.

25. Notons en passant l'homophonie "auteur/hauteur» qui nous permet de souligner que cet auteur cherche à se mettre "à hauteur " de ses personnages et de son lectorat avec lequel il entretient un rapport horizontal et interactif. 
la prégnance de la bande, traduisant un mouvement vers le lecteur dont on sollicite la participation active ${ }^{26}$. Il semble que ce mouvement soit ici particulièrement - graphiquement - mis en relief.

A l'opposé, à la page 51, la Norvégienne qui escalade la Tour Montparnasse est montrée selon un plan en "radicale plongée", ce qui fait apparaître Thomas telle une fourmi au pied de la tour: "peut-être fallait-il se réjouir de n'être plus qu'un petit point du passé qui bientôt s'effacerait totalement", dit le récitatif. De même, la première de couverture montre un héros lilliputien aux pieds d'une géante et dont le regard est tourné vers celle-ci (fig. 1). La page 66 représente une chute de notre antihéros ("La question, c'est à quoi je sers?») - dont l'image est reprise sur la page de titre alors que le final matérialise l'envol métaphorique du couple (p. 80) en un mouvement inverse d'ascension souligné par un oiseau. Il en résulte une forme de mise en scène doublée d'une mise en espace questionnant l'équilibre des rapports homme-femme, question que l'on retrouve au cœur du théâtre de Bégaudeau: d'abord dans $L e$ Problème, puis dans Un deux un deux ${ }^{27}$.

\section{3. Les mises en abyme et citations graphiques}

Dans $M O C$, le roman Belle du seigneur est graphiquement cité comme un appât pour séduire: échec cuisant, fin de non recevoir qui flirte avec le burlesque (fig. 3-4). Or cette œuvre magistrale de Cohen nous apparaît éminemment significative d'une mise en scène de l'histoire d'amour, de la théâtralité qui lui est inhérente dans ses différentes étapes, du jeu de la séduction à la passion qui s'ensuit. Le thème du livre-appât évoque déjà une mise en scène, un jeu. Si Bégaudeau reproche à ce roman sa vision misogyne de la relation amoureuse, il semble que son personnage masculin se situe dans un rapport de domination inversé, victime d'une mise en scène qui lui échappe. La planche suivante laisse entrevoir le titre du livre qu'elle est en train de lire et qu'elle lui conseille: il s'agit de $M O C$ et Thomas découvrant l'album dans l'album semble de plus en plus abattu au fil de sa lecture. L'avant-dernière page laissera apercevoir un album intitulé FOC (i. e. «Femme Occidentale Contemporaine»).

26. G. Lascaut, in B. Eizykman (éd.), Plates bandes à part, p. 33.

27. Pièce inédite, transmise par l'auteur: http://www.theatredebelleville.com/ prochainement/item/97-un-deux-un-deux 
Fig. $3-M O C$, p. 11, vignettes 5 et 6 .

Une autre scène présente une sorte de mise en abyme: une jeune femme déguisée en soubrette aborde Thomas à la terrasse d'un café et l'embrasse de façon impromptue (voir infra, fig. 7). Il s'agit en fait d'une mise en scène factice et burlesque dans le cadre d'un enterrement de vie de jeune fille, comme on le comprend à la planche suivante (p. 76). Le lecteur, par identification à Thomas, se trouve ici pris dans une forme d'illusion dramatique, au sens étymologique de «jeu» (ludus).

2. 4. L'importance du corps ou le narcissisme amoureux

Le corps revêt une importance certaine dans l'œuvre de cet auteur (voir sa pièce intitulée $L e$ Foie) qui reconnaît cependant que son théâtre est relativement dénué de gestes (entretien cité). De fait, Thomas est souvent représenté avec les mains dans les poches, ce qui illustre une difficulté à se positionner dans l'espace. Celle-ci sera verbalisée à la page 58, notre antihéros se posant en Narcisse des temps modernes face à son miroir (fig. 4) : «Je ne sais pas comment me tenir. Je ne sais pas comment me positionner. Je ne sais pas quoi faire de mon corps. Je ne sais pas où me mettre. Qu'est-ce que je vais faire de moi ?». En un mouvement réflexif, cette scène nous invite à envisager la $\mathrm{BD}$ comme miroir: "Je suis un miroir à alouettes, un miroir à humains, Je multiplie les embuscades ${ }^{28}$. Une dizaine de pages plus loin, il tentera de se travestir (p. 70) : «Moi la BD, je suis un agent double. [...] Je suis toujours un autre et je suis plurielle» 29 .

28. G. Lascault, in B. Eizykman (éd.), Plates bandes à part, p. 22.

29. Ibid., p. 21. 


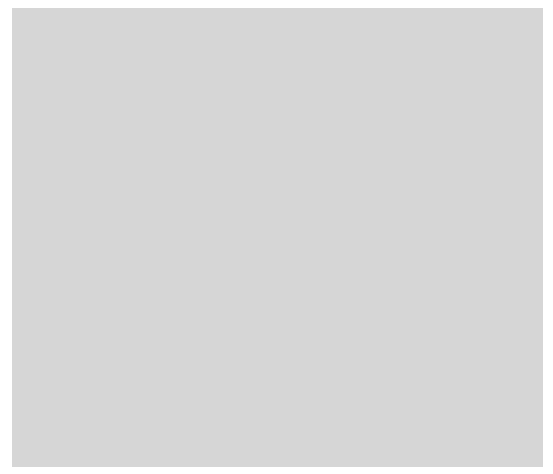

Fig. 4-MOC, p. 58, vignette 6 .

2. 5. Les banderoles ou le comique de situation littéralisé

Les slogans des manifestations nous parlent aussi du corps: «Les machos, on en a plein les couilles» clament les féministes (p. 27). "Mon clitoris m'appartient" figure ironiquement sur une banderole que Thomas brandit. (p. 46). Les banderoles se trouvent ici mises en relief par une sorte de cadre dans le cadre, qui se substitue presque aux dialogues, révélant une ironie mordante: "Je déteste les faux féministes» / "Je suis juste venu pour draguer...» (p. 27, reprise sur la page intérieure de l'album). Il s'agit là, au sens propre, d'un dialogue de sourds matérialisé par l'écrit. C'est encore les rapports homme-femme et une forme de malentendu qui sont interrogés: "Moi je dresse un inventaire des femmes. [...] Je multiplie les déboires sentimentaux sans pathos » ${ }^{30}$ (fig. 5).

2. 6. Les dialogues de sourds

Dans la BD, la plupart des dialogues - y compris en chat sur la toile, représentés dans des cadres roses et bleus comme de réels dialogues dont les locuteurs seraient hors-case (p. 53) - tournent court, virant au dialogue de sourds, voire au monologue (p. 18). Cela renvoie au motif de l'incommunicabilité, qu'on retrouve notamment dans la pièce Le Foie, ou encore du Problème. A contrario, dans la pièce Un deux un deux qui rend compte des étapes ultérieures d'une histoire amoureuse, une réelle connivence se manifestera au travers des dialogues. Il nous semble alors que la $\mathrm{BD}$, du fait de sa structure conçue comme un éternel recommencement

30. Ibid., p. 27. 
Fig. $5-M O C$, p. 18 , vignettes 5 et 6 .

que reflète la fragmentation en planches, mette au jour ce qu'on pourrait appeler un «bégaudage» (en référence au «marivaudage») qui se caractériserait par une entrée en matière aussi timide que directe et maladroite ( Pardon, je crois que je viens de tomber amoureux de vous»), souvent répétitive, dénuée de préciosité et convoquant un humour touchant au burlesque qui traverse les autres écrits de l'auteur: «Pardon, j'imite très bien le cri du pingouin». Dans le tapuscrit, la répétition du «Pardon» apparaît en gras, comme un ajout, anaphore soulignant la mécanique comique. La répétition est au service de l'humour, reflétant le fameux «mécanique plaqué sur du vivant» de Bergson. Dans cet échange, la succession des lieux que permet la $\mathrm{BD}$ à travers la succession de cases met en relief le rebond ironique de la réplique «cool!»:

L: Tranquille. J'ai un mec depuis six mois.

Lui: cool!

L: il veut qu'on fasse un enfant

Lui : cool!

Ascenseur

L: en plus on sera raccord sur l'éducation, on s'est rencontrés aux Jeunes socialistes.

Lui : cool.

Rue

$\mathrm{L}$ : on espère juste que ce sera un trisomique

Lui: cool!

Ils se quittent. 
La ponctuation exprime le désarroi mutique du héros dans certaines situations, les ponctèmes remplissant parfois l'espace d'un phylactère et se substituant alors au discours, ou le précédant en une sorte de cataphore (fig. 6).

Fig. $6-M O C$, p. 25 , vignettes 3 et 4 .

Il serait donc intéressant d'appréhender la $\mathrm{BD}$ comme un medium adéquat à évoquer la part de jeu (et de malaise potentiel) initial dans la séduction - jeu qui donne ici à rire à la façon d'une comédie - préalable à la relation en elle-même qui pourrait trouver le cas échéant un medium privilégié dans le théâtre avec le développement d'un dialogue où les voix d'Elle et Lui se conjuguent (voir la pièce Un deux un deux) alors qu'elles se cherchent - et ne se trouvent pas forcément dans la BD. Dans l'entretien qu'il nous a consacré, François Bégaudeau a insisté sur sa recherche des potentialités médiopoétiques propres à une forme/ un support: "je suis relativement plastique d'un art à l'autre parce que je me demande toujours ce que cette forme induit comme mode d'énoncé». Sémiotiquement, la BD met en relief une superposition de voix de différentes natures mais inscrites simultanément dans un même espace graphique qui entrent - ou non - en dialogue et en résonance. La fin de $M O C$ donne à voir (à entendre) un perroquet moqueur allégorisant une "voix intérieure" qui tend vers l'autodérision (fig. 7). Il va de soi que cette voix-ci - certes allégorique mais présentée comme effectivement proférée - prend une tonalité particulière, incarnée par le détour animalier qui accentue la distanciation. 


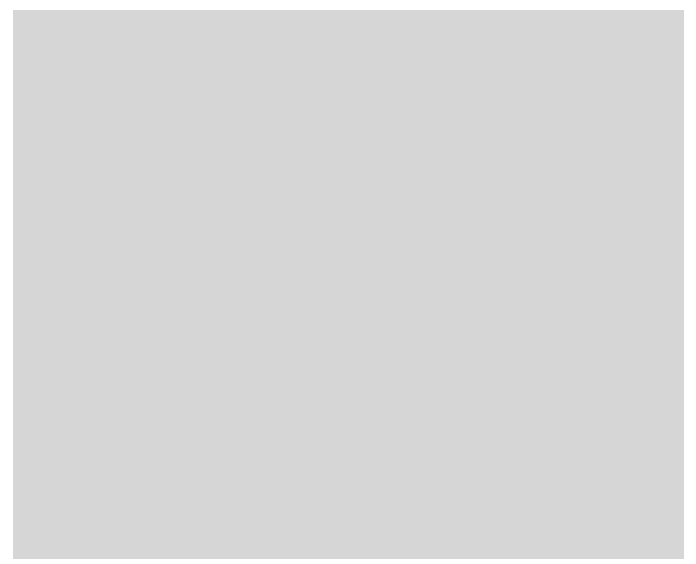

Fig. $7-M O C$, p. 76, vignette 4 .

\section{Petite frappe ou la théâtralité du foot}

3. 1. Du marivaudage au bégaudage, l'art de la drague

Lauteur a scénarisé une deuxième $\mathrm{BD}$, récemment parue sous le titre Petite frappe (avec Grégory Mardon qui l'a illustrée), mettant en scène les aventures d'un jeune footballeur (fig. 8). Dans la continuité de l'album précédent, on retrouve une forme de "bégaudage" à travers la mise en scène d'une opération de drague du protagoniste pour son ami. Marivaux n'est pas si loin:

- Y a mon pote Azdine, il t’a élue fille de l'année.

- J'suis contente pour lui.

- A égalité avec Leila Bekthi.

- Pourquoi il vient pas me le dire?

- Les résultats du vote sont encore secrets.

- Pourquoi tu les dis alors?

- Ça me fait un prétexte pour te parler (p. 27) (fig. 9).

3. 2. Théâtralité dans le monde footballistique

Ainsi, même si cette deuxième $\mathrm{BD}$ s'avère plus résolument narrative que la première qui se caractérise par un faible degré de narrativité, la théâtralité y est encore patente: le terrain de foot représente la scène alors 
Fig. 8 - Petite Frappe, première de couverture.

Fig. 9 - Petite frappe, p. 27, vignettes 1, 2 et 3 . 
que les vestiaires constituent les coulisses. La première de couverture (voir fig. 8) suggère la dimension spectaculaire et scénique du football en le reliant à la scène musicale. En outre, certaines planches illustrent parfaitement la mimogestualité dont usent et abusent les footballeurs et dont la coupe du monde nous a offert un nouvel aperçu (fig. 10). Les corps sont magnifiés ainsi que les gestes techniques, l'auteur ayant d'ailleurs coordonné un ouvrage sur le sujet ${ }^{31}$. Les planches donnent à voir une sorte de ballet (pour le dire avec les mots de Blutch, cité précédemment), de chorégraphie autour du ballon.

Outre cette mise en lumière des corps, les cadres enchâssés dans la BD - qui illustre sémiotiquement une dialectique ordre-désordre - peuvent signifier l'autorité (voire les autorités enchâssées) à laquelle cet adolescent rétif est soumis et tend à échapper. Notons que l'auteur est aussi essayiste ${ }^{32}$, critique et journaliste sportif. Il a aussi conçu et interprété une pièce sur le foot (Non réconciliés), ainsi qu'une fable étiologique (L'invention du jeu).

Fig. 10 - Petite frappe, p. 7, vignette 5.

3. 3. Eil de lynx et oreille de rocker

L'une des originalités de cette $\mathrm{BD}$ - et sans doute l'un des foyers de littérarité - est d'accueillir en son sein un lynx aux échos mythologiques et aux traits féminins, aussi allégorique qu'énigmatique. Celui-ci est

3I. Avec X. de La Porte, Le Sport par les gestes.

32. Il est l'auteur, avec Joy Sorman, d'un essai sur la jeunesse: Parce que ça nous plaît. L'invention de la jeunesse, Paris, Larousse, 2010. 
d'abord entraperçu puis rencontré par Jon au cours d'une séquence dont les couleurs rosées et violacées suggèrent l'onirisme. Il vient délivrer un message, tout en délivrant précisément notre héros d'un destin tracé. Et la $\mathrm{BD}$, au delà du jeu amoureux du chat et de la souris illustré par $M O C$, de se faire récit initiatique au travers de cette voix particulièrement éloquente (fig. 11).

En outre, un nouveau medium intervient ici à travers la musique dès lors que la $\mathrm{BD}$ est accompagnée d'une bande-son, avec des liens à suivre pour écouter les morceaux sur la toile: il s'agit de la BO de la BD. C'est l'ex-auteur et chanteur du groupe "Zabriskie point ${ }^{33}$ qui exprime son amour infaillible pour le rock en mettant en scène cette expérience à travers un groupe de rockeuses. C'est la musique qui permet métaphoriquement l'échappée, l'envol, l'émancipation, la sortie du cadre que suggère la dernière image (fig. 12), en un mouvement métaphorique d'ascension, d'envol qui n'est pas sans rappeler celui de $M O C$.

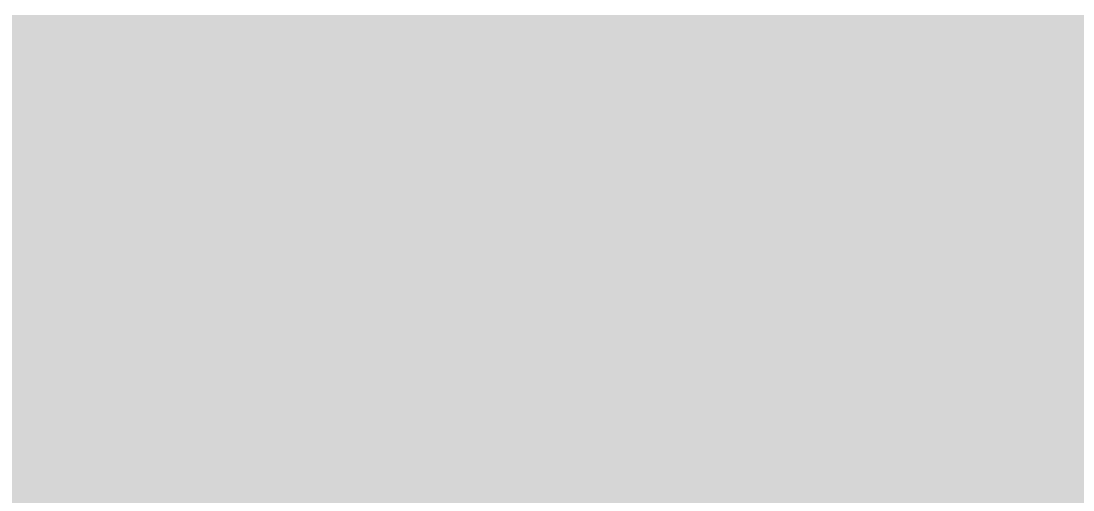

Fig. 11 - Petite frappe, p. 148, vignettes 1 et 2 .

\section{Ecrire à travers les genres}

Au vu de tous ces liens intermédiaux qu'éclaire le parcours polyvalent de l'auteur, l'œuvre de ce dernier nous apparaît emblématique du concept de médiaculture. De fait, il ne rechigne pas à aborder par la $\mathrm{BD}$ des thèmes qui lui sont chers et qu'il a traités sous d'autres formes médiales: son premier roman Jouer juste entremêlait déjà l'amour et le foot. Dans

33. Notons que ce nom de groupe fait encore référence au cinéma (film d'Antonioni). 
ces conditions, il ne semble pas y avoir de medium privilégié chez Bégaudeau qui s'essaie à tous les media et registres. D'une certaine façon, il œuvre ainsi, et notamment à travers la BD qui n'est pas "casable» (selon le bon mot de Dürrenmatt), à déconstruire la hiérarchie des genres, à déjouer les codes en interrogeant en permanence les frontières intermédiales: son écriture touche à tout, à tous les genres, y compris au sens sexuel de ce terme puisqu'il n'hésite pas non plus à adopter des points de vue féminins par exemple dans Au début (récits sur la maternité) ${ }^{34}$ ou encore à travestir son personnage comme dans $M O C$. Il lui plaît aussi d'intégrer des liens hypertextuels vers des clips musicaux, constituant par là-même une sorte de $\mathrm{BO}$ de ses livres (voir aussi Deux singes ou ma vie politique). L'intermédialité - ou plus précisément, parlons avec Jean-Pierre Bobillot, de "permédialité» avec ce que ce concept évoque de perméabilité, de porosité - apparaissent alors au cœur de son œuvre, comme en témoignent les formes multiples qu'ont pris, prennent et prendront ses travaux: théâtre, $\mathrm{BD}$, films ${ }^{35}$, pièce radiophonique, chroniques journalistiques... Notons que la dimension participative est ici nodale: l'auteur tient un site internet qui manifeste sa fibre essentiellement démocrate et constitue un prolongement, pour le coup "multimédial», de son œuvre écrite et dramaturgique, au travers duquel il anime et met en scène sa posture d'auteur ${ }^{36}$. Une posture marquée par l'humour, la recherche d'une frappe comique qui se décline différemment selon les espaces médiologiques investis: la BD rend particulièrement lisible/visible le décalage, le malentendu voire la mésentente entre plusieurs voix qui tendent à se superposer plutôt qu'à se croiser ou à se conjuguer, le malaise et la solitude des corps engendrés par certaines situations, la répétition, mise en relief par la succession des cases, d'une même situation ou d'un même schéma dialogique qui tourne en boucle. La BD nous apparait alors comme un lieu propre aux rebonds comiques comme aux rebondissements théâtraux, aux coups de théâtre, tout en offrant un espace de jeu et de subversion, d'échappée et de délivrance vis-à-vis des codes éprouvés dans le cadre du roman.

Camille Vorger Université de Lausanne

34. Texte qui a fait l'objet d'une lecture à plusieurs voix au théâtre de Dax.

35. Son roman La Blessure, la vraie est en cours d'adaptation par Kechiche et les droits de $M O C$ ont été achetés dans cette même perspective cinématographique.

36. http://begaudeau.info/ 
Fig. 12 - Petite frappe, p. 158. 


\section{BIBLIOGRAPHIE}

BARThes, Roland, Fragments d'un discours amoureux, coll. "Tel quel", Paris, Editions du Seuil, 1977.

BÉgaudeau, François, Jouer juste, Paris, éditions Verticales, 2003.

—, Entre les murs, éditions Verticales, 2006.

—, Le Problème, éditions Théâtre ouvert, 2008.

—, Le Foie, éditions Théâtre ouvert, 2012.

—, Un deux, un deux, pièce inédite (en duo avec Mélanie Mary).

—, Non réconciliés, pièce inédite.

—, «Mort et vif», Transfuge, 80 (septembre 2014), p. 8-10.

Bégaudeau, François, La Porte, Xavier de, Le sport par les gestes, Paris Calmann Lévy, 2007.

Bégaudeau, François, Lemaître, Pascal, L’invention du jeu, Paris, Hélium éditions, 2009.

Bégaudeau, François, Mardon, Grégory, Petite frappe, Paris, Delcourt/ Mirages, 2014.

Bégaudeau, François, Oubrerie, Clément, Mâle Occidental Contemporain, Paris, Delcourt/Mirages, 2013.

Bernanoce, Marie, "La didactique du texte de théâtre: comment penser la relation entre écriture et oralité? La notion de voix didascalique", in Philippe Clermont, Anne Schneider (éds), Ecoute mon papyrus. Littérature, oral et oralité, Strasbourg, SCEREN/CRDP d'Alsace, 2006, p. 225-240.

—, "Des indications scéniques à la "voix didascalique": contours énonciatifs de la figure de l'auteur de théâtre contemporain", Coulisses, 39 (2009), p. 31-42.

Dacheux, Eric, Le Pontois, Sandrine, La BD, un miroir du lien social, Bande dessinée et solidarités, Paris, L'Harmattan, 2011.

Dürrenmatt, Jacques, Bande dessinée et littérature, Paris, Classiques Garnier, 2013.

Eizykman, Boris (éd.), Plates bandes à part. Esthétique de la bande dessinée, Bruxelles, La lettre volée/Université de Picardie, 2012. 
Groesteen, Thierry, La bande dessinée mode d'emploi, Bruxelles, Impressions nouvelles, 2008.

Maigret, Eric, Macé, Eric, Penser les médiacultures, Nouvelles pratiques et nouvelles approches de la représentation du monde. Paris, Amrmand, Colin, 2005.

Maigret, Eric, Stefanelli, Matteo (éds), La bande dessinée: une médiaculture, Paris, Armand Colin/INA éditions, 2012.

Crédits iconographiques

Bégaudeau, François, Oubrerie, Clément, Mâle Occidental Contemporain.

(C) Editions Delcourt, 2013.

Bégaudeau, François, Mardon, Grégory, Petite frappe.

(C) Editions Delcourt, 2014. 
